\title{
Treatment of abdominal aorta aneurysms
}

\author{
V Gasbarro \\ From de Senectute: Age and Health Forum \\ Catanzaro, Italy. 5-7 December 2009
}

\section{Background}

The best treatment of abdominal aorta aneurysms of the segments below the emergency of the renal arteries are still under discussion. In literature there are several studies that compare the retroperitoneal (R.P.) approach with the transperitoneal (T.P.) one or that suggest their possible variations. The R.P. approach offers the possibility of treating patients with hostyle abdomen (former laparotomic surgery, obesity) or in a state of chronic respiratory insufficiency; it allows for a better exposure of the over and infra-renal abdominal aorta segments and most of all it is characterised by a better post surgery period associated with a lower number of postoperatory complications with a faster restart of the intestinal functions and with shorter hospitalization.

\section{Materials and methods}

From 1996 and 2008, 686 planned surgical treatments of the subrenal abdominal aorta have been performed. All the anamnestic, surgical and anaesthesiological data concerning all the treated patients have been collected in an electronic database. The follow-up has been carried out through phone interviews and clinical examinations, within our vascular O.U..

220 patients underwent endovascular treatment, 170 underwent surgical treatment with T.P. approach (group A) and 296 through R.P. or left lombotomic approach (group B). The patients treated in emergency or through endovascular procedures have been excluded by our study.

The T.P. group (group A) is made up by 170 patients, while the R.P. (group B) by 296 patients. The average follow-up lasts 66 months for group A and 72 for group B. Our study has not showed statistically significant differences between the two groups in relation with age, sex, aneurysm dimentions, the presence of risk factors and possible association with hypertension, coronary disease, COBP, hyperlipidemia, diabetes, chronic kidney failure, smoke addiction, while there is a significant statistical difference between the two groups regarding obesity which is more frequent in group B.

Also in our study the post surgery period of the patients treated with the R.P. approach was characterized by a faster restart of evacuation, and such a difference was statistically significant. Such a result was due to the fact that the R.P. approach did not request the opening of the peritoneum therefore reducing the damage of intestinal homeostasys.

\section{Results and conclusions}

The number of patients admitted to the intensive care unit after surgery was significantly lower in group A. Anyway the length of admission in the intensive care unit was comparable in the two groups. Moreover the patients of group B did not have statistically shorter hospitalization showing a faster restart of autonomy than the patients of group B.

The percentage of death cases within 30 days and 2 years from surgical treatment were higher in the T.P. group, such a difference was not statistically higher in group B. Our study underlined that the R.P. approach was associated with better results in terms of post surgery complications, length of admission and long term survival. Consequently this method is a first choice in our O.U. for the planned treatment of AAA.

Published: 19 May 2010

doi:10.1186/1471-2318-10-S1-A57

Cite this article as: Gasbarro: Treatment of abdominal aorta aneurysms. BMC Geriatrics 2010 10(Suppl 1):A57. 